\title{
RUNX1 Germline Mutation in a Patient with Chronic Thrombocytopenia
}

\author{
Yujin Nam, Gyu Min Yeon and Seom Gim Kong \\ Department of Pediatrics, Kosin University Gospel Hospital, Kosin University College of Medicine, Busan, Korea
}

\begin{abstract}
One of the pathophysiologic mechanism of inherited thrombocytopenia is a defect in transcription factors that regulate the expression of multiple genes required for megakaryopoiesis. Runt-related transcription factor 1 (RUNX1) binds to its heterodimeric partner, core binding factor beta $(\mathrm{CBF} \beta)$, and forms a core binding factor that regulates the expression of various target genes. The association between RUNX1 germline mutations and familial platelet disorder with associated myeloid malignancy was first reported in 1999. Although this disease has various phenotypes and penetration, the most common symptom is a bleeding tendency due to thrombocytopenia and platelet dysfunction. Myelodysplastic syndromes or acute myeloid leukemia may also develop in $35-40 \%$ of cases. We identified a heterozygous mutation in the RUNX1 gene using diagnostic exome sequencing in an adolescent with chronic thrombocytopenia. The patient will be followed continuously for hematologic malignancies that may develop in the future. This case illustrates the importance of diagnosing inherited thrombocytopenia to provide adequate follow-up for hematologic malignancies and reduce unnecessary treatment.
\end{abstract}

Key Words: RUNX1 translocation partner 1 protein, Germ-line mutation, Thrombocytopenia, Platelet disorder, familial, with associated myeloid malignancy
pISSN 2233-5250 / eISSN 2233-4580 https://doi.org/10.15264/cpho.2021.28.2.89 Clin Pediatr Hematol Oncol 2021;28:89 92

Received on September 30, 2021 Revised on October 12, 2021 Accepted on October 14, 2021

\section{Introduction}

Runt-related transcription factor 1 (RUNX1), also known as AML1 or core-binding factor subunit $\alpha-2$ $(\mathrm{CBF} \alpha 2)$, is a transcription factor that regulates the expression of genes that are necessary for the normal development of hematopoietic stem cells. RUNX1 binds to its heterodimeric partner core binding factor beta (CBF $\beta$ ) to form a core binding factor, and regulates the expression of various target genes, including hematopoietic differentiation, ribosome biogenesis, cell cycle regulation, p53 and transforming growth factor $\beta$ signaling pathways [1]. Somatic mutations in RUNX1 and translocation with other genes are well known to be asso- ciated with myelodysplastic syndromes and leukemia.

Germline mutations in RUNX1 can cause familial platelet disorders with associated myeloid malignancy (FPD/AML). FPD/AML was first reported in 1978 in a sibling family with bleeding tendencies, thrombocytopenia, and myeloproliferative disease [2]. Then, in 1999, a RUNX1 heterozygous mutation was associated with this disease, and more than 70 affected families have been reported until recently [3]. FPD/AML presents with mild to moderate thrombocytopenia and platelet dysfunction and is known to increase the risk of hematologic malignancies such as myelodysplastic syndrome (MDS) and acute myeloid leukemia (AML) [1,4]. We report a case of diagnosis of FPD/AML that was confirmed by identifying a germline mutation in the RUNX1 gene in a patient with 
chronic thrombocytopenia who had experienced and reported a bleeding tendency from an early age. This study was approved by the Institutional Review Board of our hospital (KUGH 2021-09-035).

\section{Case Report}

A 13-year-old boy presented at our clinic for persistent thrombocytopenia. Blood testing at the age of 10 found that his platelet count was $82,000 / \mu \mathrm{L}$. After that initial testing, a blood test was performed at a nearby hospital every 6 months, and the platelet count continued to be about 80,000-90,000/ $\mu$ L. Relevant birth history indicated that he was born as a full-term infant by cesarean section, and there was no specific past history. The patient had experienced frequent bruising since childhood and reported similar symptoms recently. His parents and brother did not present with signs of bleeding tendency.

The blood test performed at the first visit at the age of 10 also revealed leukocytes levels of 5,660/ $\mu \mathrm{L}$, hemoglobin $13.0 \mathrm{~g} / \mathrm{dL}$, and platelets at 83,000/ $\mathrm{LL}$. Prothrombin time and partial thromboplastin time were within the normal ranges, and the anti-nuclear antibody and anti-platelet antibody tests were negative. The von Willebrand factor (vWF) antigen test result was 69\%, the vWF ristocetin cofactor activity test was $68 \%$, and the $\beta$-glucosidase activity was $7.3 \mathrm{nmol} / \mathrm{hr} / \mathrm{mg}(6.0-9.0 \mathrm{nmol} / \mathrm{hr} / \mathrm{mg})$. C3, $\mathrm{C} 4$ and $\mathrm{CH} 50$ levels were normal, and $\mathrm{H}$. pylori antibody was also negative. Platelet function tests and bone marrow study were not performed. Thereafter, follow-up testing values for the platelet count continued indicated in the range of $47,000-87,000 / \mu \mathrm{L}$.

After follow-up, inherited thrombocytopenia was suspected because the patient continued to experience thrombocytopenia, had a more severe bleeding tendency compared to the platelet count, and had a bleeding tendency similar to that reported in childhood. Diagnostic exome sequencing was performed on the patient at the age of 18. Diagnostic exome sequencing was performed through peripheral blood, and 5,447 target genes were analyzed using a NextSeq 550 System (Illumina, San Diego,

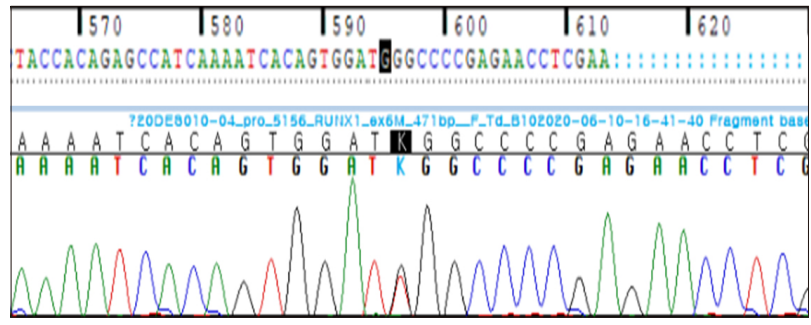

Fig. 1. DNA sequencing of RUNX1 demonstrated a G-to-T transition and predicted to result in a glycine-to-tryptophan change to the amino acid 199 (c.595G > T, p.Gly199Trp).

USA). DNA sequencing demonstrated a heterozygous mutation of the RUNX1 gene (c.595G > T, p.Gly199Trp) (Fig. 1). For missense variants, in silico analysis was performed using SIFT, PolyPhen-2, and MutationTaster, and annotation was done with VEP99 (Varient Effect Predictor), dbNSFP v3.5. This mutation was a very rare mutation that has not been reported in the general population. We performed a Sanger sequencing on the peripheral blood of the parents, and no mutations were found, so it was confirmed as a de novo mutation. The patient, at the most recent age of 20 , had a platelet count of $52,000 / \mu \mathrm{L}$, and the tendency for bruising and bleeding persists, although, he currently living without any reported problems or treatment. However, the patient requires continuous follow-up to assess development of hematologic malignancies such as AML or MDS.

\section{Discussion}

Inherited thrombocytopenias have been identified in more than 30 diseases [5]. Inherited thrombocytopenias are caused by germline mutations of genes associated with each step of the platelet production process, which can be divided into three groups [6]. The first step is differentiation and proliferation of hematopoietic stem cells into immature megakaryocytes. The second step is the maturation of immature megakaryocytes. In this stage, DNA accumulates in up to $128 \mathrm{~N}$ through endomitosis, and a large amount of mRNA and proteins stored in $\alpha$-granules are produced. The third step is the production of proplatelets and the release of platelets. Transcription factors such as RUNX1, FLI1, GATA1, 
GFI1b, and ETVG are mainly associated with the maturation of megakaryocytes. However, they act as activators or repressors for the expression of various genes that are required for megakaryopoiesis as well as megakaryocyte maturation [7].

RUNX1 is an essential gene for hematopoiesis, and research has reported that RUNX1 knockout mice died from severe bleeding during embryonic development [8]. In adult mice studies, RUNX1-deficient mice showed inefficient platelet production, abnormal B- and T-cell maturation, suppression of lymphocyte progenitor cell production, and expansion of myeloid progenitors [9]. Many recent studies have found that the RUNX1 gene down-regulates genes related to platelet production, structure, signaling and function. RUNX1 directly regulates the transcription of genes associated with megakaryocytic maturation (NF-E2), megakaryocytic cytoskeleton components (MYHO, MYLO, MYH1O), $\alpha$ - and dense granule development-related proteins (PF4, PLDN), and members of signaling pathways (ANKRD26, MPL, PRKCQ, ALOX12, PCTP) [5,7]. A study that identified downstream biological pathways of the RUNX1 gene suggested that a heterozygous RUNX1 mutation in FPD-AML patients was associated with impairment of cell proliferation, microtubule dynamics, and genomic stability [10].

The RUNX1 gene has two domains: a runt homology domain (RHD) and a transactivation domain (TAD) [11]. Most of the cases are caused by mutations in the RHD region. Most have missense, nonsense or frameshift mutations, and some large intragenic deletions or duplications have also been reported [1]. Symptoms may vary from patient to patient and commonly present with mild or moderate bleeding tendencies from childhood, which are attributable to thrombocytopenia $(20,000-134,000 / \mu \mathrm{L})$ and platelet dysfunction [12]. Platelet size is usually normal and may appear gray with some $\alpha$-granule reduction. Bone marrow is hypocellular or normocellular and dysmorphic megakaryocytes are found. Regarding platelet function, dense granule storage pool deficiency, partial $\alpha$-granule deficiency, fibrinogen receptor activation defects, and diffusion defects of glycoprotein IIb-IIIa and platelets appear to be contributing factors [12].
An important aspect for managing patients with FPD/ AML is the high incidence of hematologic malignancies (35-40\%). MDS and AML mainly occur, and T-cell acute lymphoblastic leukemia, hairy cell leukemia, and chronic myelomonocytic leukemia have also been reported [1]. According to a recent study, about $30 \%$ of hematologic malignancies in FPD/AML patients occurred before the age of 20 [13]. However, a RUNX1 mutation alone is not sufficient to cause hematologic malignancies in FPD/AML patients. Hematologic malignancies can occur when mutations of the opposite side of RUNX1 or other gene mutations such as AXSL1, CBL, CDC25C, FLT3, PHFG, SRSF2, or WT1 are present [12]. In general, it has been reported that the survival rate was lower in MDS and AML that occurred in patients with RUNX1 germline mutations [14, 15]. Therefore, to prevent the occurrence of secondary mutations in FPD/AML patients, education approaches, such as minimizing exposure to carcinogens, is required, and regular blood tests are recommended for detecting hematologic malignancies.

Inherited thrombocytopenia is a rare disease, and diseases related to early megakaryocyte formation defects or platelet formation and release defects such as congenital amegakaryocytic thrombocytopenia and MYHOrelated disease are relatively well known [6]. However, diseases related to maturation defects of megakaryocytes, such as RUNX1 mutations, have not been widely reported yet but with the recent expansion of access to genetic testing, diagnosis of these causes has become possible. Diagnosing inherited thrombocytopenia in patients with chronic thrombocytopenia has several important considerations. First, it may be mistaken for other diseases such as chronic idiopathic thrombocytopenia, and unnecessary tests or treatments may not be performed. Second, management of RUNX1, ANKRD2G, and ETVGrelated diseases is important because there is a possibility for these cases to progress into MDS or AML [12].

In conclusion, the suspicion of hereditary thrombocytopenia is necessary in patients with chronic thrombocytopenia that has persisted since childhood. If inherited thrombocytopenia is suspected, evaluation and considerations for diseases related to megakaryocyte matura- 
tion defects, such as FPD/AML, will also be needed.

\section{Conflict of Interest Statement}

The authors have no conflict of interest to declare.

\section{References}

1. Sood R, Kamikubo Y, Liu P. Role of RUNX1 in hematological malignancies. Blood 2017;129:2070-82.

2. Luddy RE, Champion LA, Schwartz AD. A fatal myeloproliferative syndrome in a family with thrombocytopenia and platelet dysfunction. Cancer 1978;41:1959-63.

3. Song WJ, Sullivan MG, Legare RD, et al. Haploinsufficiency of CBFA2 causes familial thrombocytopenia with propensity to develop acute myelogenous leukaemia. Nat Genet 1999; 23:166-75

4. Park M. Myelodysplastic syndrome with genetic predisposition. Blood Res 2021;56(S1):S34-8

5. Noris P, Pecci A. Hereditary thrombocytopenias: a growing list of disorders. Hematology Am Soc Hematol Educ Program 2017;2017:385-99.

6. Savoia A. Molecular basis of inherited thrombocytopenias. Clin Genet 2016;89:154-62.

7. Songdej N, Rao AK. Hematopoietic transcription factor mutations: important players in inherited platelet defects. Blood
2017; 129:2873-81.

8. Okuda T, van Deursen J, Hiebert SW, Grosveld G, Downing JR. AML1, the target of multiple chromosomal translocations in human leukemia, is essential for normal fetal liver hematopoiesis. Cell 1996;84:321-30.

9. Growney JD, Shigematsu H, Li Z, et al. Loss of Runx1 perturbs adult hematopoiesis and is associated with a myeloproliferative phenotype. Blood 2005;106:494-504.

10. Michaud J, Simpson KM, Escher R, et al. Integrative analysis of RUNX1 downstream pathways and target genes. BMC Genomics 2008;9:363.

11. Schlegelberger B, Heller PG. RUNX1 deficiency (familial platelet disorder with predisposition to myeloid leukemia, FPDMM). Semin Hematol 2017;54:75-80.

12. Galera P, Dulau-Florea A, Calvo KR. Inherited thrombocytopenia and platelet disorders with germline predisposition to myeloid neoplasia. Int J Lab Hematol 2019;41 Suppl 1:13141.

13. Hayashi Y, Harada Y, Huang G, Harada H. Myeloid neoplasms with germ line RUNX1 mutation. Int J Hematol 2017;106:1838.

14. You E, Cho YU, Jang S, et al. Frequency and clinicopathologic features of RUNX1 mutations in patients with acute myeloid leukemia not otherwise specified. Am J Clin Pathol 2017;148:64-72.

15. He W, Zhao C, Hu H. Prognostic effect of RUNX1 mutations in myelodysplastic syndromes: a meta-analysis. Hematology 2020;25:494-501. 